\title{
A Virtual Meal-Making Environment as a Platform to Measure the Effect of Affective Stimuli on Emotional Response and Task Performance in Children with and without Cerebral Palsy
}

\author{
Sharon Kirshner ${ }^{1} \quad$ Patrice. L. (Tamar) Weiss ${ }^{1} \quad$ Emanuel Tirosh $^{2,3}$ \\ (1)Department of Occupational Therapy, University of Haifa, Israel \\ (2) Faculty of Medicine, Technion, Israel \\ (3) Child Developmental Center, Bnai-Zion Medical Center, Haifa, Israel \\ E-mail:sharonkirshner@gmail.com
}

\begin{abstract}
The study examined whether a functional virtual environment (VE) may be used to provide affective stimuli (AS) that lead to changes in the emotional responses and task performance of children with and without cerebral palsy $(C P)$. Fifteen children with $C P$ and 19 typically developing (TD) peers (6 to 12 years) prepared seven virtual meals in a predefined order within the Emotional Meal-Maker (EMM), a virtual meal-making $V E$, run on a $2 D$ video capture $V R$ platform. Six meals included either a negative, positive, or neutral visual stimuli, selected from the International Affective Picture System (IAPS). Heart rate (HR) and skin conductance were recorded online, synchronized with stimulus onset. Significant differences were found between groups in task performance and heart rate variability (HRV) components, e.g., higher low frequency (LF)/ high frequency $(H F)$ ratio in $C P$ during the EMM task $(U=2517.5, p<001)$, regardless of type of $A S$. No significant changes in autonomic responses as a function of $A S$ were found. The implications of these results are discussed.
\end{abstract}

\section{Introduction}

Participation is defined as a person's involvement in life situations which include, for example, areas of personal maintenance, mobility, social relationships, communication, leisure, and education [1]. It is the context in which children can develop skills, create friendships, learn, perform activities, and achieve self satisfaction and a sense of competence, and is, therefore, crucial for psychological and emotional well-being as well as skill development $[2,3]$.
Children and young adults with cerebral palsy (CP) sustain primary impairments in the neuromuscular system. The motor disorders are often accompanied by disturbances of other systems such as sensation, perception, cognition, and behavior [4]. These impairments were found to be significant barriers to participation in many activity domains [2, 3, 5-7], as well as in social life and communication [8]. The influence of affective stimuli (AS) within an environment (e.g., sounds, sights) on emotion, task performance and children's participation is currently unknown, although there is evidence to suggest that their effect may be substantial at many levels $[9,10]$. Shapiro et al. [10] examined the effect of two different dental environments on the arousal level as measured by electrodermal activity in children with and without developmental disabilities. They found that a sensory adaptive environment that included special lighting effects, relaxing music, vibration, and aromas versus a conventional environment had a significantly greater calming effect on children in both groups with the decrease being most pronounced in the children with developmental delays.

Gesturetek's IREX video capture virtual reality (VR) system may be programmed to present visual and auditory stimuli that accompany virtual games and functional environments. This type of VR has been shown to provide enjoyable leisure activity for young adults with cerebral palsy and severe intellectual disabilities [11] and appears to lead to increased selfesteem, motivation [12] and sense of mastery [13]. Studies further indicate the effectiveness of VR in promoting motor control [14], upper extremity functioning [15] and cortical reorganization [16].

The goals of this study were to determine whether a functional virtual environment (VE) may be used to provide affective stimuli that lead to changes in the emotional response of a child with $\mathrm{CP}$ and in task

This is an Open Access article distributed under the terms of the Creative Commons Attribution-Noncommercial License 3.0, which permits unrestricted use, distribution, and reproduction in any noncommercial medium, provided the original work is properly cited. 
performance outcomes, and to examine the differences in emotional response and performance between children with $\mathrm{CP}$ and typically developing (TD) peers. The Meal-Maker (MM) VE was developed within IREX and modified to include the delivery of affective stimuli, referred to as the Emotional Meal-Maker (EMM). The usability of the MM and its sensitivity to performance differences between children with and without CP has been reported [17].

\section{Methods}

\subsection{Subjects}

Fifteen children with spastic CP (study group, mean $\pm \mathrm{SD}=9.3 \pm 1.4$ years) and 19 typically developing (TD) children (control group, mean $\pm \mathrm{SD}=8.9 \pm 1.6$ years) participated in the study. Children in the CP group who had Gross Motor Function Classification System (GMFCS) scores between 1 to 4, were able to reach with their dominant hand, and were able to follow multi-step instructions were included. Children who had an epileptic seizure within six months prior to the study, significant visual acuity or field deficits, hearing impairment, or any unstable medical condition were excluded from the study. Ethical approval was granted by the Institutional Review Boards of the University of Haifa, the Bnai Zion Medical Center, and the Israeli Ministry of Education.

\subsection{Emotional Meal-Maker VE}

The Emotional Meal-Maker is a modified version of the Meal-Maker VE, developed within a video-capture VR system (gesturetekhealth.com) [17]. Subjects were instructed to prepare as many repetitions as possible of seven different meals, presented in a predefined order. Each meal included 5 relevant items for meal preparation and 2 non meal distracters, all located on virtual kitchen shelves. A video camera recorded the child's gestures which thereby controlled navigation within the EMM and selection of items. Only movements made by the dominant hand which was holding a red cone were captured. Selection of the virtual meal items was accomplished by dwelling over the item for $2 \mathrm{~s}$. Selected items were transferred to a virtual table. Once all correct items were selected, the child hovered over a virtual "finish" button and positive visual and auditory feedback were provided.

Six different pictures from the International Affective Picture System (IAPS) [18, 19] were selected to evoke different emotional responses in the children. The first meal (hot chocolate) was considered as a baseline meal without any AS. Each of the remaining six meals presented a different stimulus while the sequence of the AS was randomized across subjects.

Electrocardiogram (ECG) and skin conductance level (SCL) were monitored to record changes in autonomic responses. Data acquisition was accomplished via the Biopac System. ECG signal gain was set at 1000 times amplification. Band pass for ECG was initially limited to $35 \mathrm{~Hz}$ low pass (LP) and $0.5 \mathrm{~Hz}$ high pass (HP), with a further $2 \mathrm{~Hz} \mathrm{HP}$ filter applied post acquisition resulting in a $2 \mathrm{~Hz}$ to $35 \mathrm{~Hz}$ band. The biological signal of the SCL was DC coupled filtered by LP set at $10 \mathrm{~Hz}$ with a further $3 \mathrm{~Hz}$ LP post acquisition. The amplification was set to $5 \mu$ Siemens/volt. The data were sampled at $1000 \mathrm{~Hz}$ and stored on a computer running Acqnowledge 3.9.1 MP100 data acquisition software.

ECG Biopac EL 501 electrodes were placed over the lateral aspect of the left and right intercostal space between either the eighth and ninth ribs or ninth and tenth ribs, in a straight line below the axila. This placement was selected to maintain uniform skin contact without disturbance by upper arm and trunk movement. The ground electrode was initially placed about $1 \mathrm{~cm}$ above the medial malleolus of the left leg, however due to spasticity related movement of the legs observed in children with CP during the EMM, the position of the ground electrode was changed to the left aspect of the lower back.

Tonic skin conductance level (SCL) measured in microSiemens $(\mu \mathrm{S})$ was recorded via Biopack TSD203 SCL transducer filled with isotonic electrogel placed over the volar surface of the middle phalanges of the second and third finger of the non-dominant hand. AC-coupled skin conductance fluctuations (skin conductance response, SCR) were analyzed offline using AcqKnowledge 4.1 software by Biopac systems Inc. The phasic signal from the tonic SCL was done using a smoothing baseline removal method.

The EMM was synchronized with the Biopac data acquisition system using a voice-recognition marker, separating the stereo sound signal of each EMM virtual item to enable marker detection and AS presentation. Based on recommendations by the Heart Rate Variability (HRV) task-force [20] the onset of the EMM marker was followed by a 2 min sampling period in order to collect sufficient HRV data for analysis. The children's cooperation during this period was encouraged by instructing them to prepare as many repetitions of each meal as they could within the 2 min period.

Online performance measures recorded during the EMM included number of correct and incorrect repetitions for each meal, total meal preparation time, and percent success (total number of correct repetitions divided by the total number of meals). 


\subsection{Procedures}

After written parental consent was given, a child and parent were invited to a $90 \mathrm{~min}$ session in the Laboratory for Innovations in Rehabilitation Technology (LIRT) in the department of Occupational Therapy at the University of Haifa, Israel. The room was air-conditioned and illuminated to establish a pleasant setting that was also sufficient and uniform for the video capture system and physiological recordings. Children initially completed two questionnaires (State/Trait Anxiety and Short Sensory Profile). Then they were introduced to IREX VR through a virtual game (Birds \& Balls) for 2 min and performed the MM VE followed by a 5 min break.

ECG and skin conductance were monitored during three conditions: calm-state, EMM, and a PowerPoint presentation (PPT); an initial 2 min steady-state period was collected during which the children were instructed to sit calmly with their eyes closed while listening to a series of statements designed to relax them. Two conditions were then presented in a counter-balanced order - the EMM task and a PPT task. The PPT included AS that were displayed in the same sequence as presented in the EMM, however it required only viewing the $\mathrm{AS}$ rather than moving to make meals as in the EMM task. As in the EMM, the time-interval between each AS presented within the PPT was $2 \mathrm{~min}$, and was accompanied by narrated stories from the "Grimm Brothers" tales in the same order and similar intonation for all children. Valence and arousal for each AS were recorded using the SelfAssessment Manikin (SAM) [21].

Subjective responses to the task within VR were recorded for both MM and EMM via Short Feedback Questionnaire, child version (SFQ-Child).

\subsection{Data Reduction and Analysis}

RR intervals were identified via the MP100 and manually checked and verified. Time domain analysis (RMSSD, NN50, pNN50) as well as frequency domain analysis (peak frequencies, total powers, normalized powers and $\mathrm{LF} / \mathrm{HF}$ ratio) were analyzed offline to measure the change in HRV via Kubios-HRV software version 2.00. Tonic SCL was measured for each task including the calm-state. Log transformation was carried out to normalize the distribution. In order to examine the change in SCL between conditions with AS versus baseline conditions (no stimuli conditions presented in both calm-state and during EMM-hot chocolate meal) the difference between Log SCL during the PPT task and the calm-state was calculated, while within EMM task the difference between the log SCL of the first AS conditioned meal (omelet) versus
Hot chocolate (baseline meal) was calculated. SCR amplitude ( $\mu$ Siemens) was scored as the largest change relative to baseline within 1 to $6 \mathrm{~s}$ after stimulus onset. SCR responses were included in the analysis only if the SCR was at least $0.01 \mu$ Siemens. The square root (SQRT) of all SCR responses were calculated in order to reduce the skewness of the distribution [22].

The Statistical Packages for the Social Sciences (SPSS) version 15 was used for statistical analysis. Due to non-normal distributions of many of the physiological and performance variables nonparametric tests were used to examine changes as a function of AS type within groups and to examine differences in physiology and performance between groups. Alpha was set at 0.05 . Correlations of behavioral questionnaires (anxiety, sensory profile) and SAM ratings with both autonomic responses and performance variables were also calculated; however these will not be presented in this paper.

\section{Results}

\subsection{Examining change in physiology and performance as affected by AS}

No significant differences were observed in autonomic responses within the $\mathrm{CP}$ group. However, within the TD group both the peak frequencies HF and the absolute power of VLF during the PPT task $(0.31 \pm$ $0.06 \mathrm{~Hz} ; 883.58 \pm 780.34 \mathrm{~ms}^{\wedge} 2$, respectively) were significantly higher than their values during the calmstate (peak HF: 0.28 \pm 0.05; Absolute power VLF: $\left.423.62 \mathrm{~ms}^{\wedge} 2, \mathrm{U}=99.00, \mathrm{p}<0.05\right)$. Comparing the autonomic responses while preparing a meal without any AS (hot chocolate meal) versus the calm-state baseline demonstrated for the TD group a borderline significant difference in the normalized powers of LF and normalized powers of $\mathrm{HF}(\mathrm{U}=101.0, \mathrm{p}=0.054)$ and in LF:HF ratio $(\mathrm{U}=100.5, \mathrm{p}=0.052)$. Furthermore, within the TD group the total time taken to prepare meals during the presentation of negative stimuli $(151.3 \pm 16.7 \mathrm{~s})$ was significantly shorter than the time taken to prepare meals during neutral stimuli (158.6 \pm 14.6 s) $(\mathrm{U}=498.5, \mathrm{p}<0.05)$. No significant changes were found in SCR amplitudes as a function of stimulus type for either group.

\subsection{Differences between groups}

Significant differences were found between children with CP versus TD in both components of HRV and task performance, presented in all EMM mealconditions. In general, children with $\mathrm{CP}$ had significantly higher heart rate $(103.4 \pm 12.1 \mathrm{bpm})$ than 
TD group (92.2 \pm 9.9 bpm) $(\mathrm{U}=3041.5, \mathrm{p}<0.001)$, lower RMSSD $(31.2 \pm 19.5 \mathrm{~ms})$ versus TD (46.6 \pm $21.5 \mathrm{~ms})(\mathrm{U}=3332.0, \mathrm{p}<0.001)$, and significantly higher LF:HF ratio in CP group $(3.1 \pm 2.5)$ versus TD group (1.8 \pm 1.5$)(\mathrm{U}=3666.5, \mathrm{p}<0.0001)$ during AS meal conditions compared to the initial meal (hot chocolate) with no AS. Similar significant differences were also recorded in the calm-state and the PPT task. No significant differences in SCR amplitude were found between groups. The increased HR in the CP group was negatively correlated with the number of total and correctly prepared meals $(\mathrm{R}=-0.48, \mathrm{p}<0.05$; $\mathrm{R}=-0.39$. $\mathrm{p}<0.05$ respectively). Furthermore, with regard to EMM performance outcomes, children with $\mathrm{CP}$ were significantly slower than TD peers and prepared significantly fewer correct and overall numbers of meals.

\section{Discussion}

Engagement of children with $\mathrm{CP}$ in activities of daily living is often limited $[2,3,5,6]$ due, in part, to intrinsic factors such as motor and cognitive impairments. However, environmental factors and the contexts underlying functional performance should be carefully looked at as having important contribution to participation and independence [1, 9]. Thus, understanding the influence of affective sensory stimuli within the environment on children with special needs, such as cerebral palsy, is of great value for clinical practice and home settings.

The results showed significant differences in autonomic responses between children with CP versus typically developing peers, e.g., higher heart rate in children with CP during all AS conditions and at rest, lower RMSSD and higher LF: HF ratio. The results are supported by the study of Bjelakovic et al. [23] who examined long-term ( 24 hours) time domain components of HRV in 35 infants with central coordination disorder (16 of them were later diagnosed as having (P) and 37 age and gender matched controls. They found significantly lower SDNN, SDANN, and RMSSD parameters in the experimental group compared to typically developing infants. Other studies that examined differences in HRV components between children with and without CP during supine and standing positions [24, 25] further found significantly higher LF:HF ratio in children with CP versus healthy control subjects during supine position, indicating greater sympathetic autonomic predominance in HR than healthy controls. The researchers suggested that children with $\mathrm{CP}$ have greater energy expenditure due to muscular tone alterations, involuntary movements, and insufficient use of muscles during compensatory muscle activity, resulting in additional energy consumption [25].

The current study further found significant differences in task performance between groups in that the children with $\mathrm{CP}$ performed the EMM task significantly slower than their TD peers, prepared fewer correct meals and fewer total meals, supporting our previous work [17].

Within the TD group there was a significant change in total time in the presence of neutral stimuli versus negative ones. Studies in adults have shown that affective stimuli can capture and divert attention regardless of their relevance to the ongoing activity [26], and, therefore, may affect both cognitive functions as well as behavior [26, 27]. Since neutral stimuli had a greater impact on performance than did the negative stimuli, it is recommended to further examine the potential of cultural bias regarding the context of the IAPS. It may be that the neutral pictures were not perceived as such by the children in this study. The valence and arousal of the IAPS needs to be further investigated.

To conclude, the meal-making VE was shown to be a feasible platform for the investigation of the physiological characteristics of children with and without $\mathrm{CP}$ and how functional performance is influenced by affective stimuli. Further studies are needed to develop additional measurements of emotional responses and to refine the types of affective interference.

\section{References}

[1] World Health Organization. International Classification of Function, Disability and Health: Short version, Geneva, World Health Organization, 2001.

[2] B. Engel Yeger, T. Jarus, D. Anaby, and M. Law. Differences in patterns of participation between youths with cerebral palsy and typically developing peers, The American Journal of Occupational Therapy, vol. 63(1), pages. 96-104, Jan/Feb. 2009.

[3] M. Law, G. King, S. King, M. Kertoy, P. Hurley, P. Rosenbaum, N. Young, and S. Hanna. Patterns of participation in recreational and leisure activities among children with complex physical disabilities, Developmental Medicine and Child Neurology, doi: 10.1017/S0012162206000740, vol. 48, pages. 337-342, May 2006.

[4] P. Rosenbaum, N. Paneth, A. Leviton, M. Goldstein, M. Bax, D. Damiano, B. Dan, and B. Jacobsson. A report: the definition and classification of cerebral palsy April 2006, Developmental Medicine Child Neurology [Supplement], ProQuest Health and Medical Complete, vol. 109, pages. 8-14, Feb. 2007. 
[5] C. Imms. Children with cerebral palsy participate: a review of the literature, Disability and Rehabilitation, Informa Healthcare, doi: 10.1080/09638280701673542, vol. 30 (24), pages. 1867-1884, Dec. 2008.

[6] A. Majnemer, M. Shevell, M. Law, R. Birnbaum, G. Chilingaryan, P. Rosenbaum, and C. Poulin. Participation and enjoyment of leisure activities in school-aged children with cerebral palsy, Developmental Medicine and Child Neurology, ProQuest Health and Medical Complete, doi: $10.1111 /$ j.1469-8749.2008.03068.x, vol. 50 (10), pages. 751-758, Oct. 2008.

[7] J. Parkes, N. McCullough, and A. Madden. To what extent do children with cerebral palsy participate in everyday life situations?, Health Social Care in the Community, Blackwell Publishing Ltd., doi: 10.1111/j.1365-2524.2009.00908.x, vol. 18(3), pages. 304-315, May 2010.

[8] J. Voorman, A. Dallmeijer, C. Schuengel, D. Knol, G. Lankhorst, and J. Becher. Activities and participation of 9- to 13-year-old children with cerebral palsy, Clinical Rehabilitation, SAGE Publications, doi: $10.1177 / 0269215506069673$, vol. 20, pages. 937-948, Nov. 2006

[9] S. Mihaylov, S. Jarvis, A. Colver, and B. Beresford. Identification and description of environmental factors that influence participation of children with cerebral palsy, Developmental Medicine and Child Neurology, doi: $10.1017 / \mathrm{S} 0012162204000490$, vol. 46, pages. 299304, May 2004.

[10] M. Shapiro, H. Sgan Cohen, S. Parush, and R. Melmed. Influence of adapted environment on the anxiety of medically treated children with developmental disability, The Journal of Pediatrics, doi: 10.1016/j.jpeds.2008.10.017, vol. 154(4), pages. 546550, Apr. 2009.

[11] P.L.T. Weiss, P. Bialik, and R. Kizony. Virtual reality provides leisure time opportunities for young adults with physical and intellectual disabilities, CyberPsychology \& Behavior, Mary Ann Liebert, Inc., doi: 10.1089/109493103322011650, vol. 6, pages. 335-342, Jun. 2003.

[12] K. Harris, and D. Reid. The influence of virtual reality play on children's motivation, The Canadian Journal of Occupational Therapy, ProQuest Health and Medical Complete, vol. 72(1), pages. 21-29, Feb. 2005.

[13] D. Reid. Benefits of a virtual play rehabilitation environment for children with cerebral palsy on perceptions of self-efficacy: a pilot study, Pediatric Rehabilitation, vol. 5(3), pages. 141-148, Jul-Sep. 2002.

[14] C. Bryanton, J. Boss, M. Brien, J. McLean, A. McCormick, and H. Sveistrup. Feasibility, motivation, and selective motor control: virtual reality compared to conventional home exercise in children with cerebral palsy, CyberPsychology \& Behavior, Mary Ann Liebert,
Inc., doi: 10.1089/cpb.2006.9.123, vol. 9(2), pages. 123128, Apr. 2006.

[15] D.T. Reid. The use of virtual reality to improve upperextremity efficiency skills in children with cerebral palsy: a pilot study, Technology and Disability, IOS Press, vol. 14, pages. 53-61, 2002.

[16] S. You, S. Jang, Y. Kim, Y. Kwon, I. Barrow, and M. Hallett. Cortical reorganization induced by virtual reality therapy in a child with hemiparetic cerebral palsy, Developmental Medicine and Child Neurology, doi: 10.1017/S0012162205001234, vol. 47, pages. 628-635, Sep. 2005.

[17] S. Kirshner, P.L. Weiss, and E. Tirosh. Meal-Maker: a virtual meal preparation environment for children with cerebral palsy, European Journal of Special Needs Education, Routledge: Taylor \& Francis Group, vol. 26(3), pages. 323-336, Aug. 2011.

[18] P.J Lang, M.M. Bradley, and B.N Cuthbert. International Affective Picture System (IAPS): Instruction Manual and Affective Ratings, Technical Report A-6, The Center for Research in Psychophysiology, University of Florida, 2005.

[19] M.H. McManis, M.M. Bradley, W.K. Berg, B.N. Cuthbert, and P.J. Lang. Emotional reactions in children: verbal, physiological, and behavioral responses to affective pictures, Psychophysiology, Cambridge University Press, vol. 38(2), pages. 222-231, Mar. 2001.

[20] Task Force. Heart rate variability: Standards of measurement, physiological interpretation, and clinical use, Task Force of the European Society of Cardiology and the North American Society of Pacing and Electrophysiology, European Heart Journal, vol. 17, pages. 354-381, Mar. 1996.

[21] M.M. Bradley, and P.J. Lang. Measuring emotion: the Self-Assessment Manikin and the Semantic Differential, Journal of Behavior Therapy and Experimental Psychiatry, Pergamon, doi: 10.1016/00057916(94)90063-9, vol. 25(1), pages. 49-59, Mar, 1994.

[22] R.J. Hempel, J.H.M. Tulen, N.J.M. van Beveren, P.G.H. Mulder, and M. Hengeveld. Subjective and physiological responses to emotion-eliciting pictures in male schizophrenic patients, International Journal of Psychophysiology, Elsevier, doi: 10.1016/j.ijpsycho.2007.01.008, vol. 64(2), pages. 174183, May, 2007.

[23] B. Bjelakovic, S. Ilic, L. Dimitrijevic, B. Milovanovic, G. Kostic, L. Bjelakovic, and S. Lukic. Heart rate variability in infants with central coordination disturbance, Early Human Development, Elsevier, doi: 10.1016/j.earlhumdev.2010.01.022, vol. 86(2), pages. 77-81, Feb. 2010.

[24] E. Park, C. Park, S. Cho, J. Lee, and E. Kim. Assessment of autonomic nervous system with analysis of heart rate variability in children with spastic cerebral 
palsy, Yonsei Medical Journal, vol. 43(1), pages. 65-72, Feb. 2002.

[25] A.R. Zamuner, A.B. Cunha, E. De Silva, A.P. Negri, E. Tudella, and M.A. Moreno. The influence of motor impairment on autonomic heart rate modulation among children with cerebral palsy, Research in Developmental Disabilities, doi: 10.1016/j.ridd.2010.09.020, vol. 32(1), pages. 217-221, Jan-Feb. 2011.

[26] F. Dolcos, and G. McCarthy. Brain systems mediating cognitive interference by emotional distraction, The Journal of Neuroscience, doi: 10.1523/JNEUROSCI.5042-05.2006, vol. 26(7), pages. 2072-2079, Feb. 2006.
[27] K.S. Blair, B.W. Smith, D.G.V. Mitchell, J. Morton, M. Vythilingam, L. Pessoa, D. Fridberg, A. Zametkin, E.E. Nelson, W.C. Drevets, D.S. Pine, A. Martin, and R.J.R. Blair. Modulation of emotion by cognition and cognition by emotion, NeuroImage, Elsevier, vol. 35, pages. 430440, Mar. 2007. 